\title{
Estimation of Parameters in Geotechnical Backanalysis - II. Application to a Tunnel Excavation Problem
}

\author{
A. Gens, A. Ledesma \& E. E. Alonso \\ Geotechnical Engineering Department, ETS Ingenieros de Caminos, Technical University of Catalonia \\ (UPC), c/ Gran Capitán s/n, 08034 Barcelona, Spain
}

(Received 9 August 1994; revised version received 27 January 1995; accepted 30 January 1995)

\section{$A B S T R A C T$}

A general statistical framework to perform backanalysis in geotechnical problems from field instrumentation has been presented in a companion paper. Here, an application to a real case involving the excavation of a tunnel in stiff overconsolidated clay is described. Both, extensometer and inclinometer measurements are used as input data and elastic moduli of the ground and the value of the $K_{0}$ coefficient are estimated. The finite element method is used as the computational procedure to solve the direct problem, and has been coupled to the identification algorithm as described in the companion paper. In addition, a discussion on the reliability of the parameters identified is presented.

\section{INTRODUCTION}

In a companion paper [1], a maximum likelihood approach for solving backanalysis problems in geomechanics has been presented. In this paper, that formulation is applied to a real case, involving the identification of parameters in a tunnel excavation problem.

Within the framework of a specified model, the problem of estimation of parameters is equivalent to the minimization of a suitable objective function [1]. The expression of this function depends on the identification criterion selected. If a probabilistic framework is used, the maximum likelihood formulation provides a consistent scheme to obtain the solution of the problem. In that case, the objective function can be written as:

$$
J=\left(\mathbf{x}^{*}-\mathbf{x}\right)^{\mathrm{t}} \mathbf{C}_{\mathbf{x}}^{-1}\left(\mathbf{x}^{*}-\mathbf{x}\right)
$$


where

$\left(\mathbf{x}^{*}-\mathbf{x}\right)=$ the vector of differences between measured and computed values of some "state variables", using a fixed model

$\mathbf{C}_{\mathbf{x}}=$ the measurements covariance matrix, which represents the structure of the error measurements.

Note that in eqn (1) $\mathbf{x}=M(\mathbf{p})$, where $\mathbf{p}$ is the vector of parameters of the model $M$. Hence, the objective function depends on the parameters vector, $J=J(\mathbf{p})$, through the dependence of measurements on parameters established by the model.

The definition of the measurements covariance matrix as well as the minimization algorithms (coupled with the finite element method) available for this kind of problems have been presented before [1].

To demonstrate the applicability of the procedure to a real case, the estimation of parameters from field measurements obtained during the excavation of an urban tunnel near Barcelona (Spain) is described herein. Three different geological layers could be distinguished, and they were modelled as linear isotropic elastic materials, due to the small magnitude of the displacements measured. The Young's moduli of the three materials $\left(E_{1}\right.$, $E_{2}$ and $E_{3}$ ) and $K_{0}$ (the ratio of horizontal to vertical in situ total stress in the excavation zone) have been estimated.

In addition, the information provided by the maximum likelihood formulation is used in order to make an error analysis and to study the reliability of the parameters identified. Also, a brief comparison is made between estimated parameters from field data and those determined using laboratory tests. Finally, a prediction of the displacements in a section of the tunnel, using the parameters identified from a previous section, is performed and compared with observations.

\section{DESCRIPTION OF THE PROBLEM}

The selected field case refers to the excavation of a $200 \mathrm{~m}$ long tunnel in tertiary clay in Terrassa (Barcelona, Spain). A typical section is shown in Fig. 1, where the three geological layers involved in the problem can be seen. From top to bottom:

Layer 1. Between 4 and $6 \mathrm{~m}$ of artificial fill.

- Layer 2. From 10 to $15 \mathrm{~m}$ of stiff overconsolidated tertiary clay.

- Layer 3. Base layer of stiff clay containing abundant Miocene slate blocks.

Three sections were instrumented using two extensometers and two inclinometers in each one. The first section (A) corresponds to the origin of 

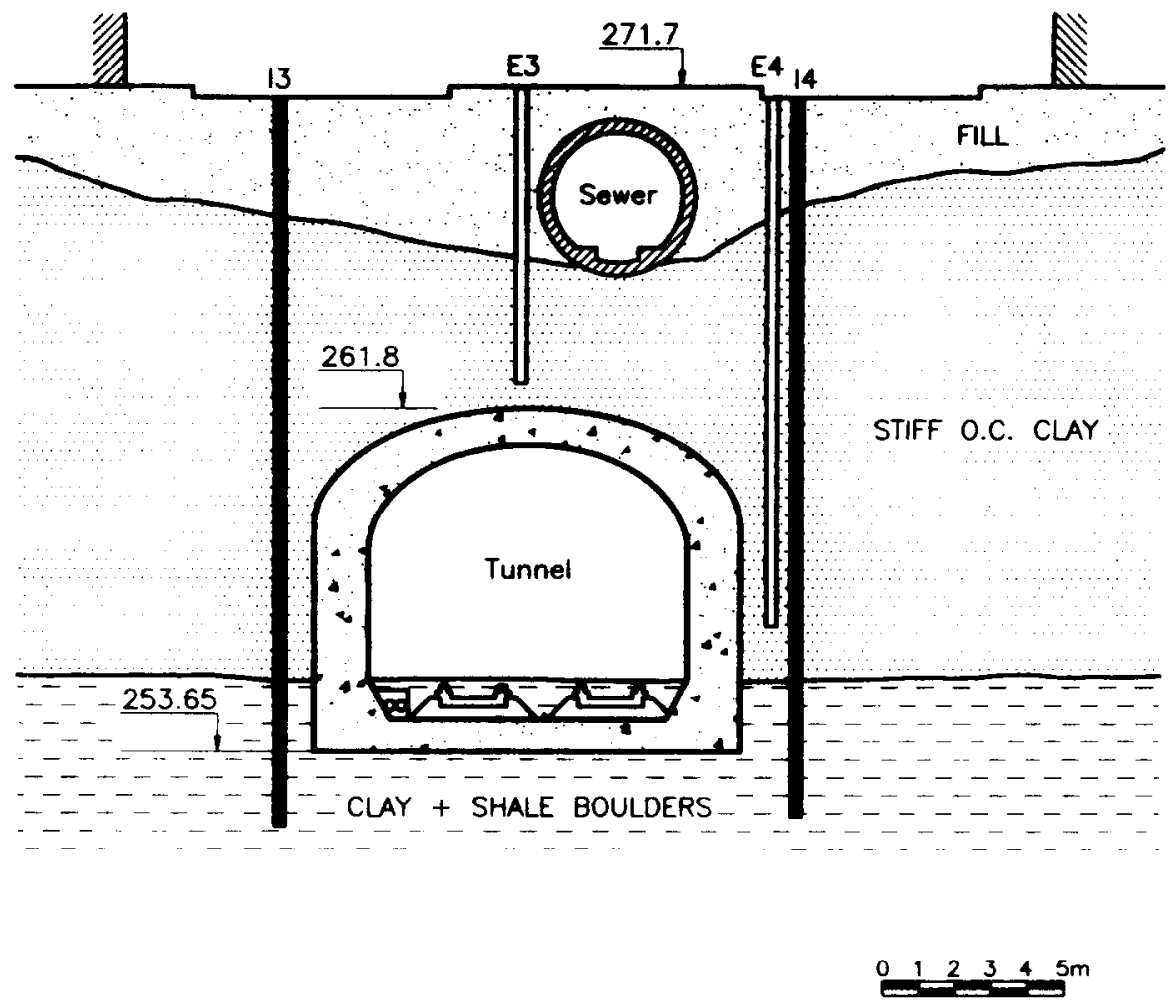

Fig. 1. Geological layers and instrumentation of tunnel section B.

the tunnel and is not easy to analyse, due to its complex geometry and to the special excavation process used. The second section (B) is the one depicted in Fig. 1, and it has been used to perform the basic identification analysis. The third section (C) was used to compare with the predictions made using the parameters estimated in section B.

The field instrumentation installed in section B (Fig. 1) was:

- one extensometer E3 on the tunnel crown,

- one extensometer E4 close to the sidewall of the tunnel,

- two inclinometers I3, I4, close to the sidewalls of the tunnel.

In order to solve the identification problem, a model relating measurements (vertical and horizontal displacements) to the set of geotechnical parameters must be specified. In this case the isotropic linear elastic model was adopted to characterize the behaviour of the three materials involved in the problem. Therefore the Young's moduli of three layers (from top to bottom: $E_{1}, E_{2}$ and $E_{3}$ ) and the $K_{0}$ parameter (ratio of horizontal to vertical in situ total stress in the excavation zone) are selected as the set of parameters 
to be identified. Poisson's ratio, $\nu$, was fixed in the analysis in order to concentrate the identification process on the main unknowns: soil stiffness and $K_{0}$ value. Although excavation was relatively fast, soil could not be assumed incompressible due to its unsaturated state (degrees of saturation of the excavated material measured in the laboratory were close to $60 \%$ ). Therefore a value of $\nu=0.3$ was selected.

The directions of initial stresses are taken as horizontal and vertical. Vertical stress was taken as $\gamma z$, where $\gamma$ is the unit weight $\left(20 \mathrm{kN} / \mathrm{m}^{3}\right)$ and $z$ the depth. The horizontal initial stress was assumed to be $K_{0} \gamma z$, where $K_{0}$ is considered as constant and unknown.

Although the excavation was carried out in several stages, only the final displacements have been introduced as input data. As a linear elastic model is used, the direct problem can be solved simulating only one excavation phase and assuming plane strain conditions. It should be recognized that the stiffness eventually identified will be higher than the real one, since the effect of the presence of the lining at the final stages of the excavation is not considered. However, such effect is probably not large since movements appear to be close to stabilizing by the time the lining was placed.

The finite element mesh used for discretization is shown in Fig. 2. In fact, on the hypothesis that the material is linear elastic, the position of the boundaries changes the solution of nodal displacements. To avoid that, relative displacements can be used as measurements in general. However, in this particular case, the boundaries were fixed taking into account the geological data available and information from section A, and finally absolute displacements were introduced in the analysis. The finite element program

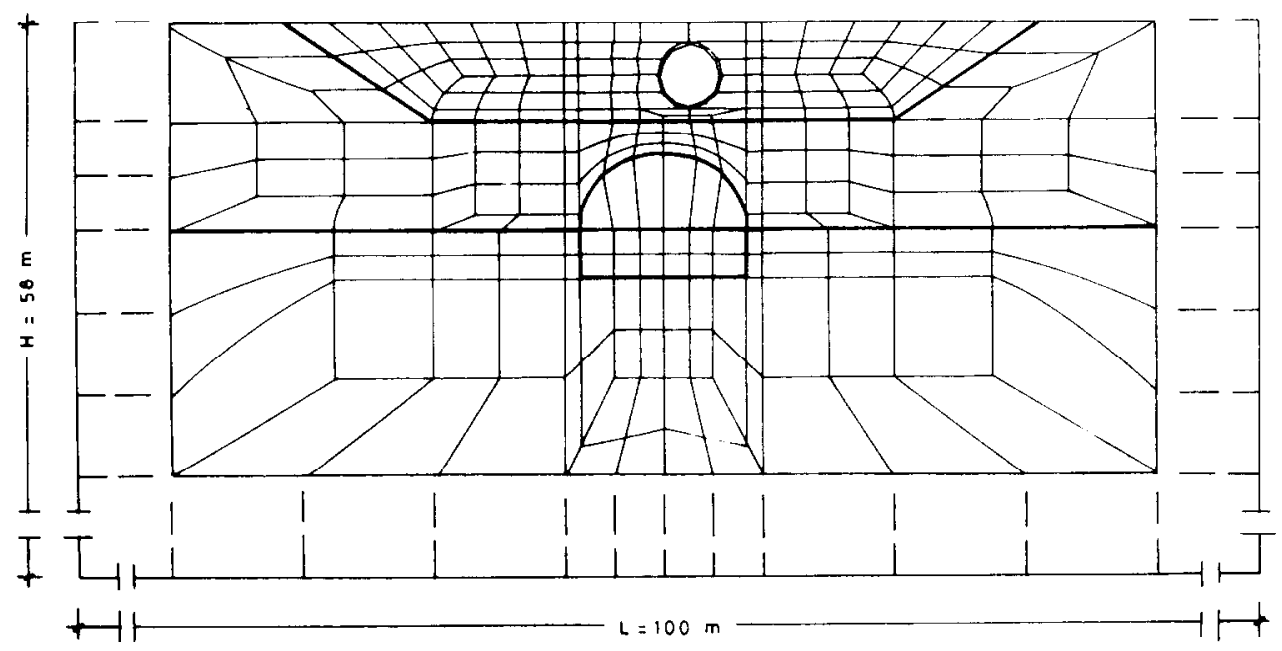

Fig. 2. Finite element mesh used in the analysis. 


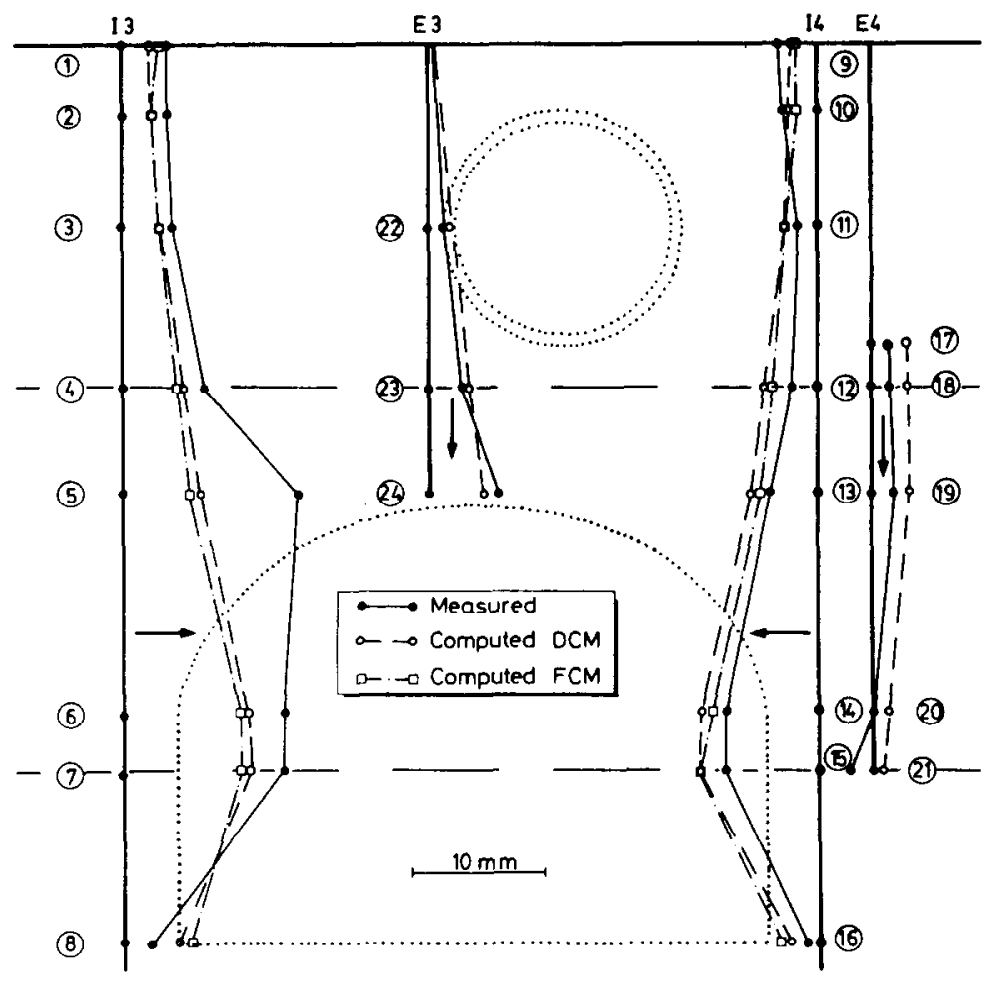

Fig. 3. Measured and computed displacements in section B. Least squares (DCM) and maximum likelihood (FCM) analyses.

"ICFEP", developed at Imperial College (U.K.), was used in the analysis. The same mesh was used in all computations.

Displacements at 24 points were used as input data, 16 were horizontal movements and 8 vertical ones. The position of the measurement points along the inclinometers and extensometers (which coincide with nodal mesh points), as well as the displacements measured, are shown in Fig. 3.

It may be seen that the maximum vertical movement is on the crown of the tunnel $(5.2 \mathrm{~mm})$ and the maximum horizontal one is around the sidewall of the tunnel $(13.1 \mathrm{~mm})$. The spatial distribution of the horizontal movements pose a difficult problem on the identification procedure since they are not the same in both inclinometers, whereas the model is basically symmetrical.

\section{LEAST SQUARES IDENTIFICATION}

As a particular case of the maximum likelihood approach, the least squares criterion can be used introducing $\mathbf{C}_{\mathbf{x}}=\sigma^{2} \mathbf{I}$ in eqn (1), where $\sigma^{2}$ is the 
measurements variance and $I$ is the identity matrix. Thus the objective function is proportional to $\sigma^{2}$ and therefore the position of the minimum of the function is not affected by this value. Then the function to be minimized is:

$$
J=\left(\mathbf{x}^{*}-\mathbf{x}\right)^{\mathrm{t}}\left(\mathbf{x}^{*}-\mathbf{x}\right)=(\boldsymbol{\Delta} \mathbf{x})^{\mathrm{t}}(\boldsymbol{\Delta} \mathbf{x})
$$

It can be shown that

$$
\Delta \mathbf{x}=\mathbf{A} \Delta \mathbf{p}
$$

where $\mathbf{A}$ is the sensitivity matrix defined in [1] (derivatives of the displacements with respect to the parameters) and $\Delta \mathbf{p}$ is the correction of the parameter values determined in the latest iteration.

Note that in a maximum likelihood framework, the least squares criterion assumes the measurements as independent and with the same variance, which is not correct in this problem, due to the use of the inclinometers.

Using the numerical procedure shown in [1] to minimize eqn (2) the following parameters have been obtained:

$$
E_{1}=98 \mathrm{MPa}, E_{2}=309 \mathrm{MPa}, E_{3}=407 \mathrm{MPa}, K_{0}=2.17
$$

The iterative process is shown in Fig. 4. It was started from a set of parameters quite different from the expected real values:

$$
E_{1}=E_{2}=E_{3}=5 \mathrm{MPa}, K_{0}=0.5
$$

and it is apparent that convergence is achieved after only 9 iterations.

The displacements computed at the measurement points using this set of parameters are shown in Fig. 3. It can be observed that the predicted horizontal movements are an "average" between the displacements measured at the two inclinometers, and that measurement 5 is practically disregarded. Vertical movements are closely matched by the computed values.

The residual value of the objective function was $1.33 \times 10^{-4} \mathrm{~m}^{2}$ and remained constant after several further iterations. This value, as it is different from 0 , could be seen as a measure of the error made using the field instrumentation, assuming the model is fixed. An estimate of the error can be obtained from the computation of the average standard deviation of the measurements, using the expression ([2]):

$$
\sigma_{x}=\sqrt{\frac{J}{m-n}}
$$




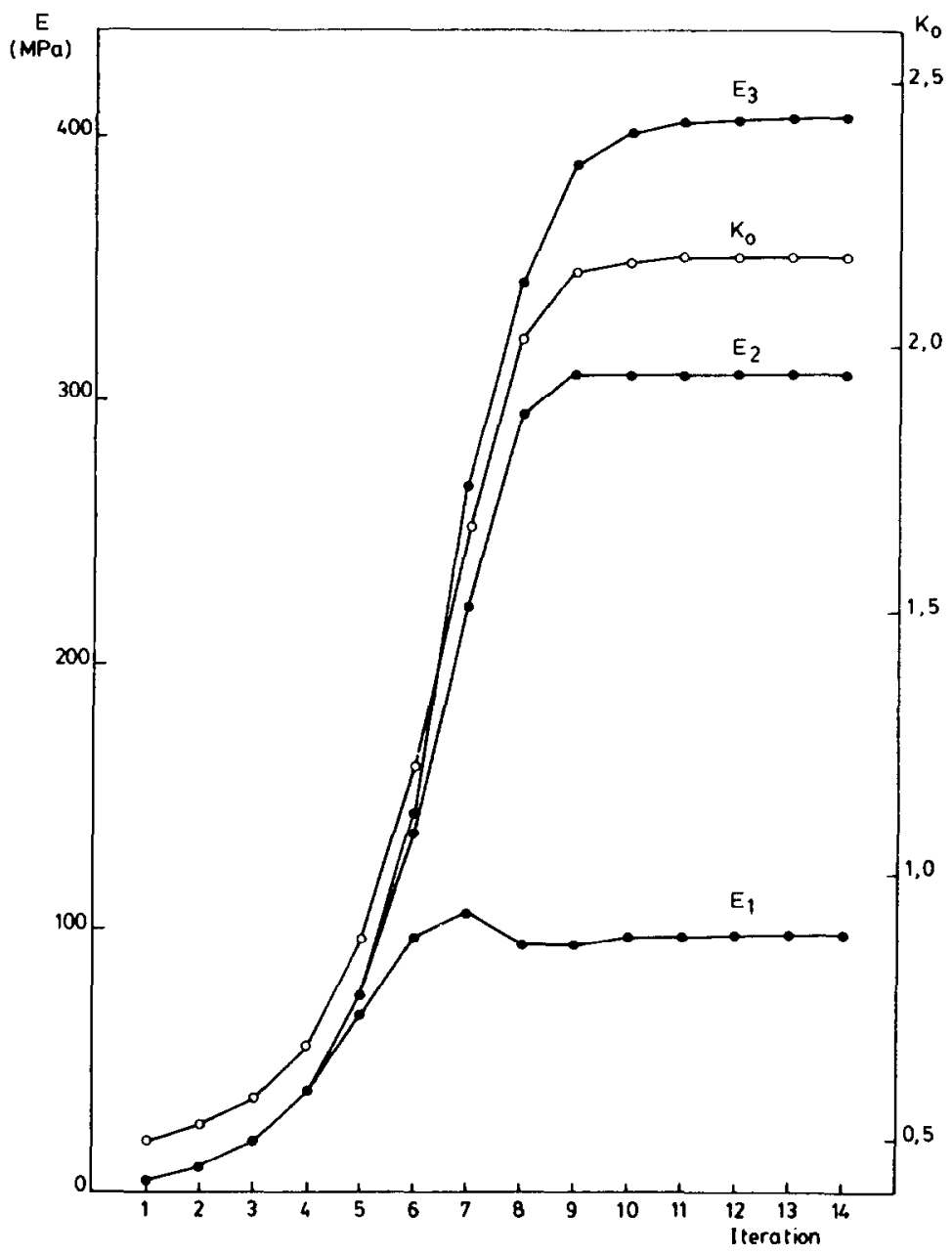

Fig. 4. Evolution of the parameters during the iteration process. Least squares analysis.

where $m$ is the number of measurements and $n$ the number of parameters involved in the identification process. In this case, $\sigma_{x}=2.58 \mathrm{~mm}$ results. As the model has been fixed in the process, the error is automatically assigned to the measurement procedure. In fact, this value is not only due to the error measurements, but also includes the differences between the model used and the actual behaviour of the materials.

The identification procedure described in [1] also allows the examination of the reliability of the parameters estimated, basically using the decomposition of the sensitivity matrix, $\mathbf{A}$, in the minimum of $J$. In Table 1 , the eigenvalues and eigenvectors of the singular decomposition of matrix $\mathbf{A}$, corresponding to the parameters space, are shown. It may be seen that the 
TABLE 1

Results of the "Least Squares" Analysis (Diagonal Covariance Matrix $\mathbf{C}_{\mathbf{x}}=\sigma^{2} \mathbf{I}$ )

\begin{tabular}{lcccc}
\hline Eigenvalues & \multicolumn{4}{c}{ Eigenvectors } \\
\cline { 2 - 5 } & $E_{l}$ & $E_{2}$ & $E_{3}$ & $K_{0}$ \\
\hline $1.370 \times 10^{-2}$ & 0.0128 & -0.1640 & -0.2058 & 0.9647 \\
$6.475 \times 10^{-3}$ & -0.6758 & -0.7156 & 0.1583 & 0.0786 \\
$1.241 \times 10^{-3}$ & -0.7265 & 0.6128 & -0.3072 & 0.0483 \\
$0.947 \times 10^{-3}$ & -0.1240 & 0.2925 & 0.9155 & 0.2467 \\
Parameters & $98 \mathrm{MPa}$ & $309 \mathrm{MPa}$ & $407 \mathrm{MPa}$ & 2.17 \\
Variances $/ \sigma_{x}^{2}$ & 0.37 & 0.35 & 0.99 & 0.07 \\
$\left(10^{2} \mathrm{MPa} / \mathrm{mm}^{2}\right)$ & & & & $25.8 \mathrm{MPa}$ \\
Standard deviation & $15.7 \mathrm{MPa}$ & $15.3 \mathrm{MPa}$ & $\mathrm{mm}^{-2}$ \\
& & & & 0.7 \\
\hline
\end{tabular}

minimum eigenvalue is closely related to $E_{3}$, whereas the maximum eigenvalue is associated with the $K_{0}$ parameter. These relationships can explain the variances of the parameters identified. However, as was indicated in [1], these relations depend also on the particular value of the parameters, so no general rule for a particular problem can be formulated. Note that in Table 1, 100 MPa has been used as a unit for the Young's moduli, in order to get similar numerical values of the $K_{0}$ parameter and to allow suitable comparisons. The final standard deviations have been estimated using the value $\sigma_{x}=2.58 \mathrm{~mm}$ as the average measurement error.

Information about the structure of the model and the interdependence of measurements can be obtained from the information density matrix $\mathbf{Q}$ [1]. Selected rows of this matrix corresponding to horizontal and vertical movements are depicted in graphical form in Fig. 5. The symmetry of the model is clearly reflected and the interdependence between vertical and horizontal movements is relatively weak. In particular, the vertical movements of extensometer E3 are quite independent from the other measurements.

One of the most interesting features of the backanalyses formulations is that parameters difficult to determine, such as $K_{0}$, can be estimated in a systematic way. To explore this capability of the procedure further, a series of identification runs were performed in which $K_{0}$ was fixed and $E_{1}, E_{2}$ and $E_{3}$ were identified. Figure 6 shows the minimum value of the objective function, $J$, for the various values of $K_{0}$ used. This is, indeed, a non-planar section of the objective function of the full identification case. It can be seen that there exists a range of $K_{0}$ in which $J$ almost do not change. This is consistent with the variance of $K_{0}$ indicated earlier, and with the fact that a range of values rather than a particular value is in fact identified. The Young's moduli identified in the $K_{0}$ fixed runs are shown in Fig. 7. Note the unreasonable 


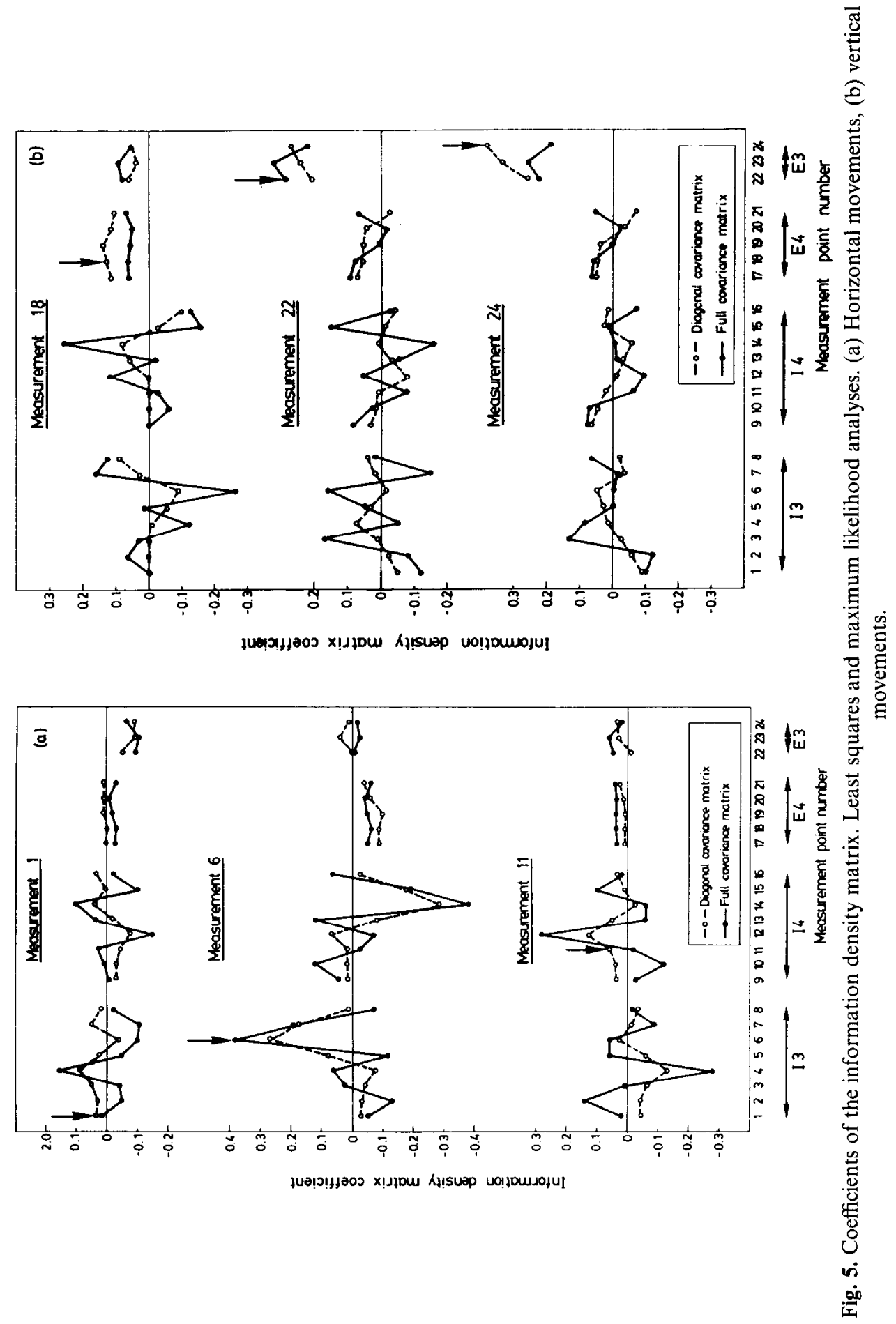




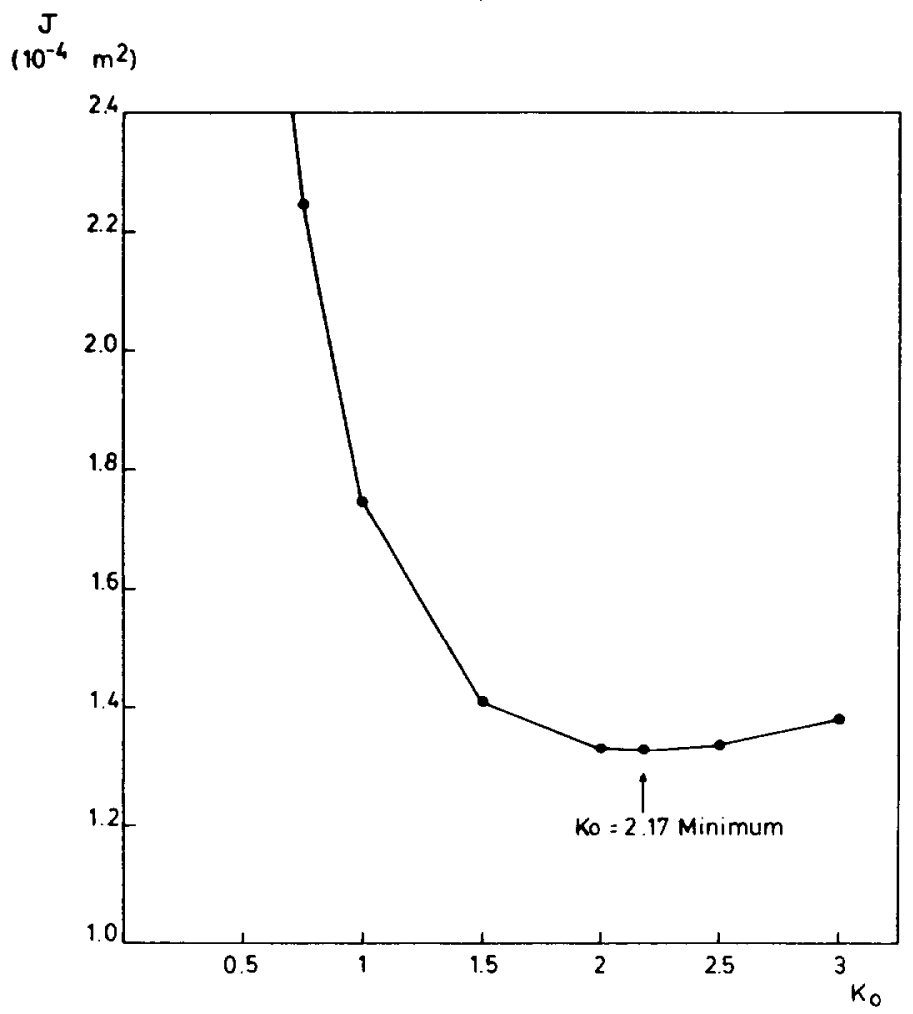

Fig. 6. Variation of the objective function with respect to $K_{0}$. Least squares analysis.

stiffness values given by the identification procedure when the value of $K_{0}$ fixed differs widely from the range of likely results. Smaller variations of $E$ are observed over the range in which a close fit to the observations is achieved.

It has been checked that variations of $\nu$ over the range of plausible values $0.2-0.4$ change the parameters identified by less than $20 \%$.

\section{MAXIMUM LIKELIHOOD IDENTIFICATION}

Taking into account the error structure of the field instrumentation, the maximum likelihood approach generalizes the objective function used in the last section to the expression:

$$
J=\left(\mathbf{x}^{*}-\mathbf{x}\right)\left(\mathbf{C}_{\mathbf{x}}^{-1}\right)\left(\mathbf{x}^{*} \quad \mathbf{x}\right)
$$

as shown in [1]. 


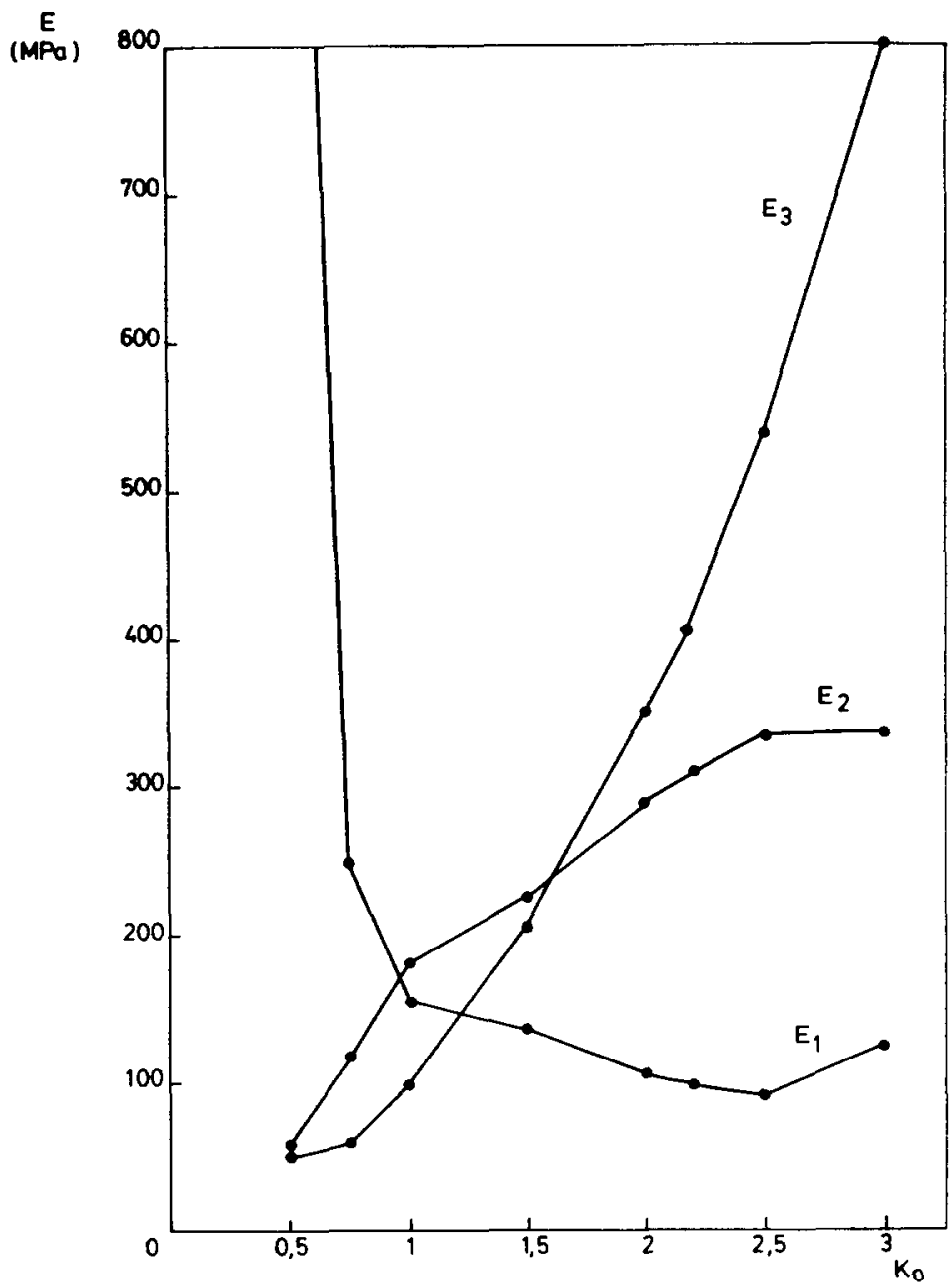

Fig. 7. Young's moduli identified for different values of fixed $K_{0}$. Least squares analysis.

Due to the use of inclinometers in this excavation problem, the measurements covariance matrix will not be diagonal, and the full covariance matrix in a complete formulation should be used:

$$
\left(\mathbf{C}_{\mathbf{x}}\right)_{I}=\sigma_{I}^{2} \sum_{m=1}^{\min (i, j)} l_{m}^{2}
$$

where $\left(\mathbf{C}_{\mathbf{x}}\right)_{I}$ is the covariance matrix of the inclinometer, as shown in [1], $\sigma_{I}^{2}$ is the variance of the slope measurement performed with the inclinometer device and $l_{m}$ is the distance between two consecutive measurement points. 
The extensometer has a diagonal covariance matrix, given that the measurements that it provides are independent and have the same variance $\sigma_{E}^{2}$. That is: $\left(\mathbf{C}_{\mathbf{x}}\right)_{E}=\sigma_{E}^{2} \mathbf{I}$.

It must be noted that performing identification analysis with full covariance matrices has been more difficult because of convergence problems in the minimization algorithm. In these cases, Marquardt's algorithm [3] had to be used. Even then, it was often found that the algorithm converged to points which did not correspond to the true minimum, especially when a very high or low value of $\sigma_{E}^{2}$ was used. This behaviour can be explained considering that a high value of $\sigma_{E}^{2}$ implies the formulation only uses the horizontal movements as input data, and there is less information available. Similar problems can occur when a low $\sigma_{E}^{2}$ is used, which implies that only vertical movements are accurate.

The case in which the same variance has been assigned to the vertical measurement and to the elemental horizontal measurement (inclinometer slope observation), has been taken as a basic solution of the maximum likelihood approach for this problem. That is $\sigma_{I}^{2}-\sigma_{t}^{2}$. The parameters identified in this case were

$$
E_{1}=134 \mathrm{MPa}, E_{2}=264 \mathrm{MPa}, E_{3}=278 \mathrm{MPa}, K_{0}=1.72
$$

However, in order to know the effect of the variances on the parameters identified, a set of identification processes using different values for $\sigma_{I}^{2}$ and $\sigma_{E}^{2}$ has been performed. It must be pointed out that the minimization of eqn (7) depends in this case on the ratio $\sigma_{I}^{2} / \sigma_{E}^{2}$ rather than on its absolute values. Figure 8 shows the parameters identified using $\sigma_{I}^{2}=1$ and different values for $\beta-1 / \sigma_{E}^{2}$. It may be observed that only $E_{3}$ and $K_{0}$ have significant dependence on the value of $\beta$.

The variances of the parameters obtained in each case are presented in Fig. 9 , normalized to an average value $\sigma_{m}^{2}=\left(8 \sigma_{E}^{2}+16 \sigma_{I}^{2}\right) / 24$ which takes into account that 8 vertical movements and 16 horizontal displacements are used in the analysis. The possibility of considering the variance ratio as a new parameter to identify, so it can be included in the identification process, has been considered elsewhere [4], and is not discussed here.

Table 2 shows the eigenvalues and eigenvectors as well as the variances of this new set of parameters estimated, corresponding to the basic case, $\beta=1$. Note that the variances of $E_{1}$ and $E_{2}$ have increased, and the variance of $E_{3}$ has decreased from the variances obtained in the least squares analysis. This change may be due to the higher reliability assigned to the deeper measurements by the inclinometer covariance matrix. The value of $K_{0}$ is in the range expected, showing an overconsolidated behaviour of the clay involved in the excavation problem. On the other hand, the gap between $E_{2}$ and $E_{3}$ is smaller 


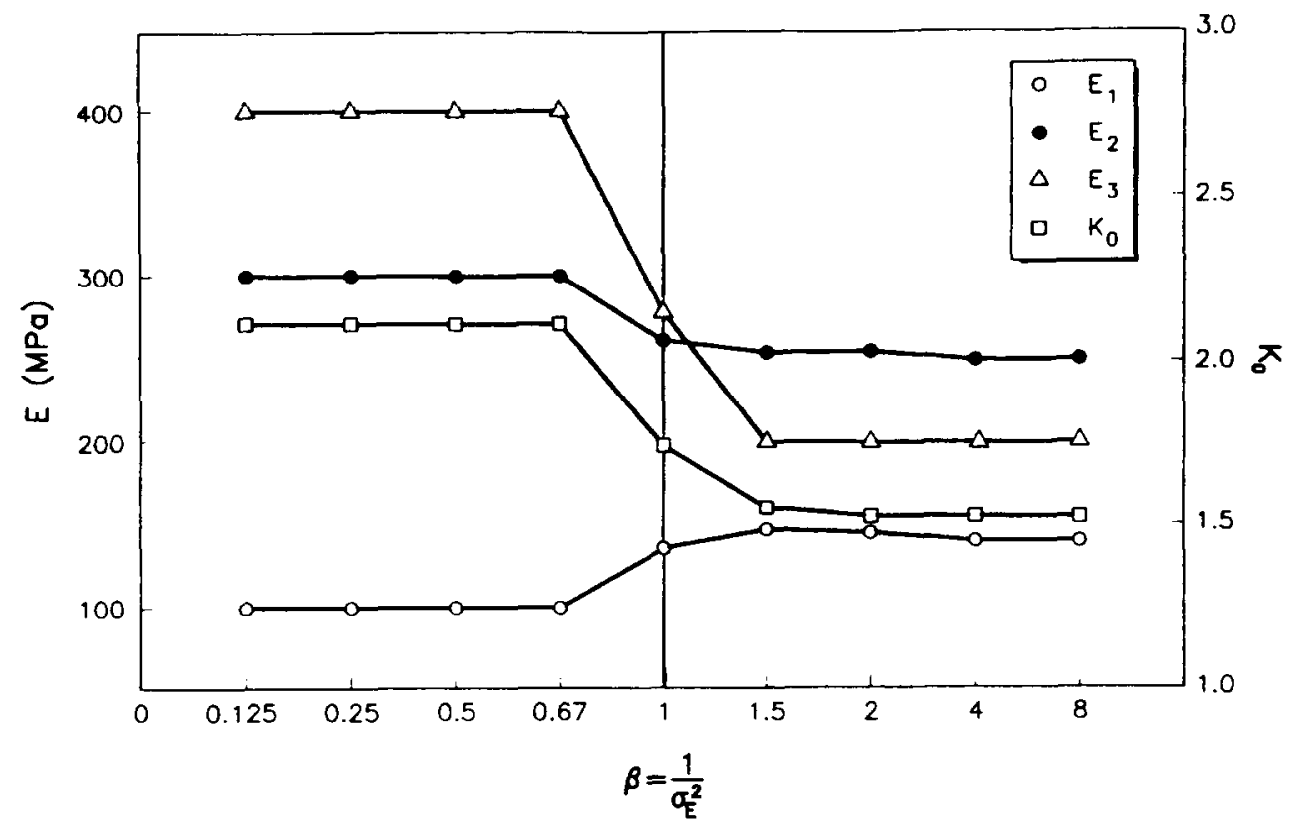

Fig. 8. Parameters estimated in the maximum likelihood analyses using different measurements covariance matrices.

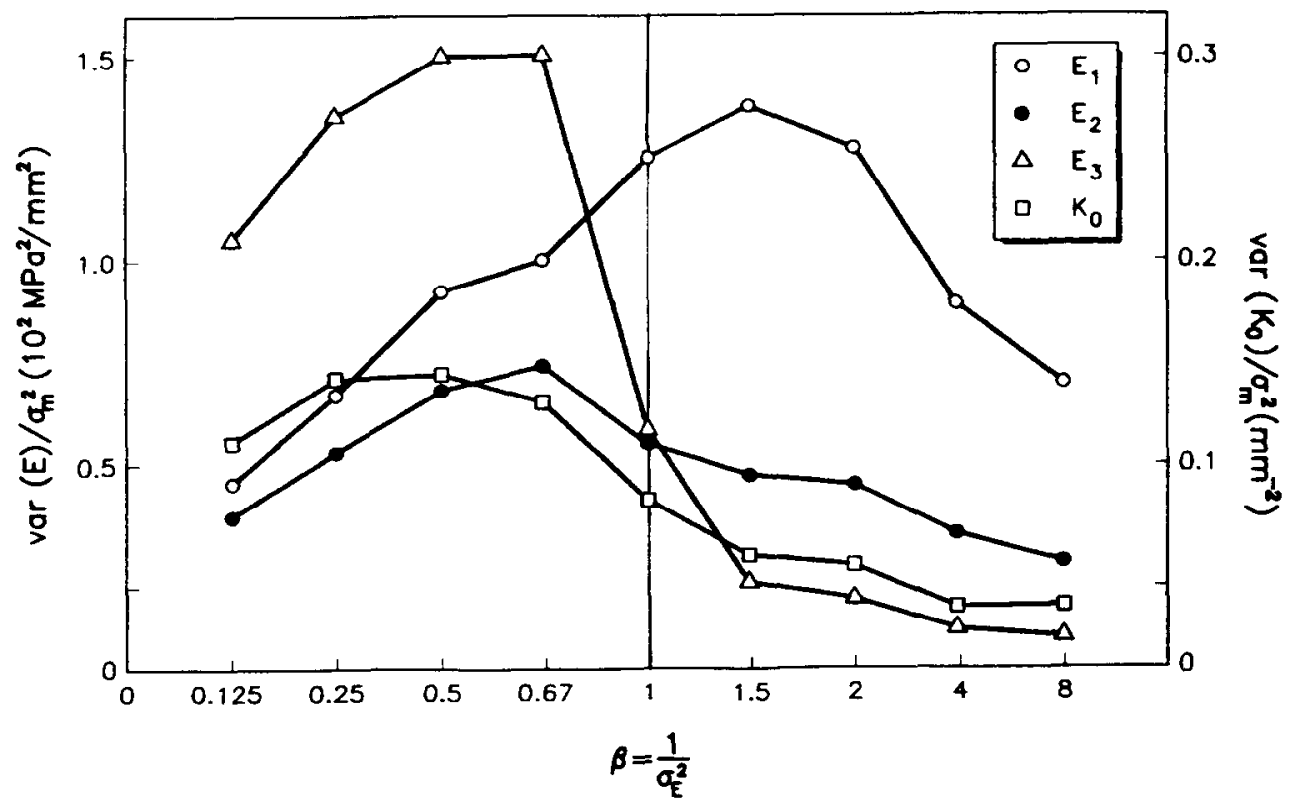

Fig. 9. Variances of the parameters estimated in the maximum likelihood analyses using different measurements covariance matrices. 
TABLE 2

Results of the "Maximum Likelihood" Analysis (Full Covariance Matrix)

\begin{tabular}{lcccc}
\hline Eigenvalues & \multicolumn{4}{c}{ Eigenvectors } \\
\cline { 2 - 5 } & $E_{l}$ & $E_{2}$ & $E_{3}$ & $K_{0}$ \\
\hline $9.642 \times 10^{-3}$ & 0.2993 & 0.2992 & -0.3141 & 0.8499 \\
$4.013 \times 10^{-3}$ & 0.3599 & -0.7700 & 0.2200 & 0.4789 \\
$1.257 \times 10^{-3}$ & 0.3544 & 0.1835 & 0.9053 & 0.1452 \\
$7.515 \times 10^{-4}$ & 0.8097 & -0.5328 & -0.1824 & -0.165 \\
Parameters & $134 \mathrm{MPa}$ & $264 \mathrm{MPa}$ & $278 \mathrm{MPa}$ & 1.72 \\
Variances $/ \sigma_{x}^{2}$ & 1.25 & 0.56 & 0.58 & 0.08 \\
$\left(10^{2} \mathrm{MPa} / \mathrm{mm}^{2}\right)$ & & & & $19.5 \mathrm{Mpa}$ \\
Standard deviation & $29 \mathrm{MPa}$ & $19.5 \mathrm{MPa}$ & & 0.73 \\
\hline
\end{tabular}

in the maximum likelihood approach, which reflects the fact that there was not too much difference between these two layers, from a geological point of view.

The average error estimated from eqn (6), using the value of the objective function in the minimum, is $2.6 \mathrm{~mm}$, similar to the value obtained in the least squares identification. The standard deviations in Table 2 have been computed using this result.

Some rows of the information density matrix have been presented in a graphical form in Fig. 5, together with those obtained in the least squares analysis (diagonal covariance matrix analysis). The general structure of the model and the importance of each group of data do not change significantly.

The displacements obtained computing the direct problem with the set of parameters (9) have been drawn in Fig. 3. There are not too many differences in the spatial distribution of the displacements calculated using the least squares parameters or the maximum likelihood parameters. It is felt however that the maximum likelihood formulation provides a more consistent framework, to consider the reliability of the parameters in terms of its variance.

\section{COMPARISON WITH LABORATORY RESULTS}

As an independent check it is interesting to compare the estimated parameters with alternative values obtained in laboratory tests. This is not always feasible: in fact, in this case, it was not possible to get samples from layers 1 (fill) and 3 (clay and slate blocks) which are highly heterogeneous at small and medium scales. So, the estimation of parameters from field instrumentation was almost the only way to obtain information about the mechanical properties of these two materials. 
However, some samples were obtained from layer 2 (overconsolidated clay) and simple compression tests with accurate measure of deformations were carried out in two specimens. The number of tests performed were not enough to use the experimental results as main information, but they were used to check the information provided by the identification procedure.

The results of these two tests are plotted in Fig. 10. One of the samples was horizontal and the other one was vertical. Slopes corresponding to Young's moduli $E_{2}$ obtained from the least squares analysis and maximum likelihood analysis are also depicted. In spite of the differences between the actual stress-strain behaviour and the linear elastic law considered in the identification analysis, the agreement can be considered satisfactory.

The experimental curves also show that there is not a high degree of anisotropy in the material, which supports the isotropic model used in the analysis. Nevertheless, experimental results show also the non-linear behaviour of the material. This should require the formulation of the proposed backanalysis procedure with a non-linear constitutive law for the material, but in this case the computation of the sensitivity matrix becomes

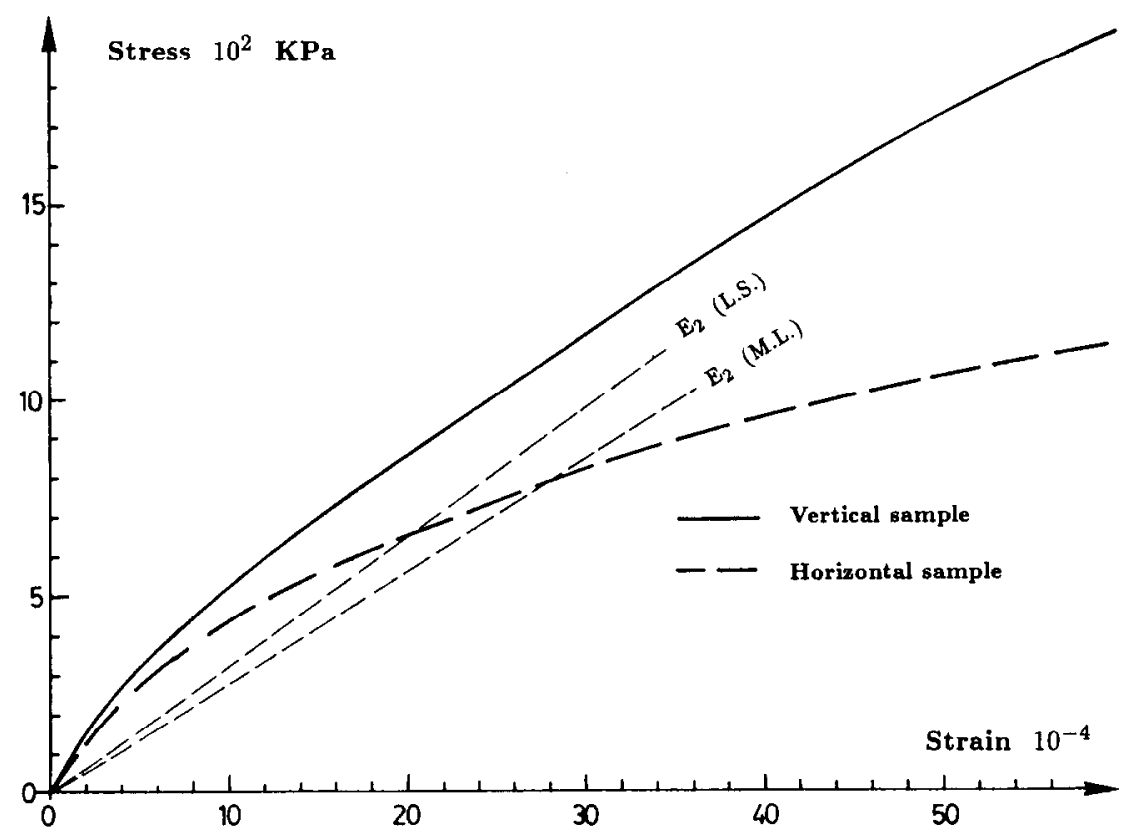

Fig. 10. Stress-strain relationships obtained in simple compression tests of clay from layer 2. Slopes of the equivalent elastic moduli from the least squares (LS) and the maximum analyses (ML). 
more difficult and the number of parameters for identification increases significantly.

\section{PREDICTION OF MOVEMENTS}

The parameters obtained from the identification problem were used to predict the movements of other sections of the tunnel still to be excavated, so that possible damages to nearby structures could be avoided using remedial measures if necessary. As an example, the prediction for section $\mathrm{C}$, which involves excavation only in the second layer and has a deeper cover depth, is herein considered. In that section, two extensometers (E5, E6) and two inclinometers (I5, I6) were installed as shown in Fig. 11.

The values of measured and predicted displacements calculated using both sets of parameters - eqns (4) and (9) - are also shown in Fig. 11. The

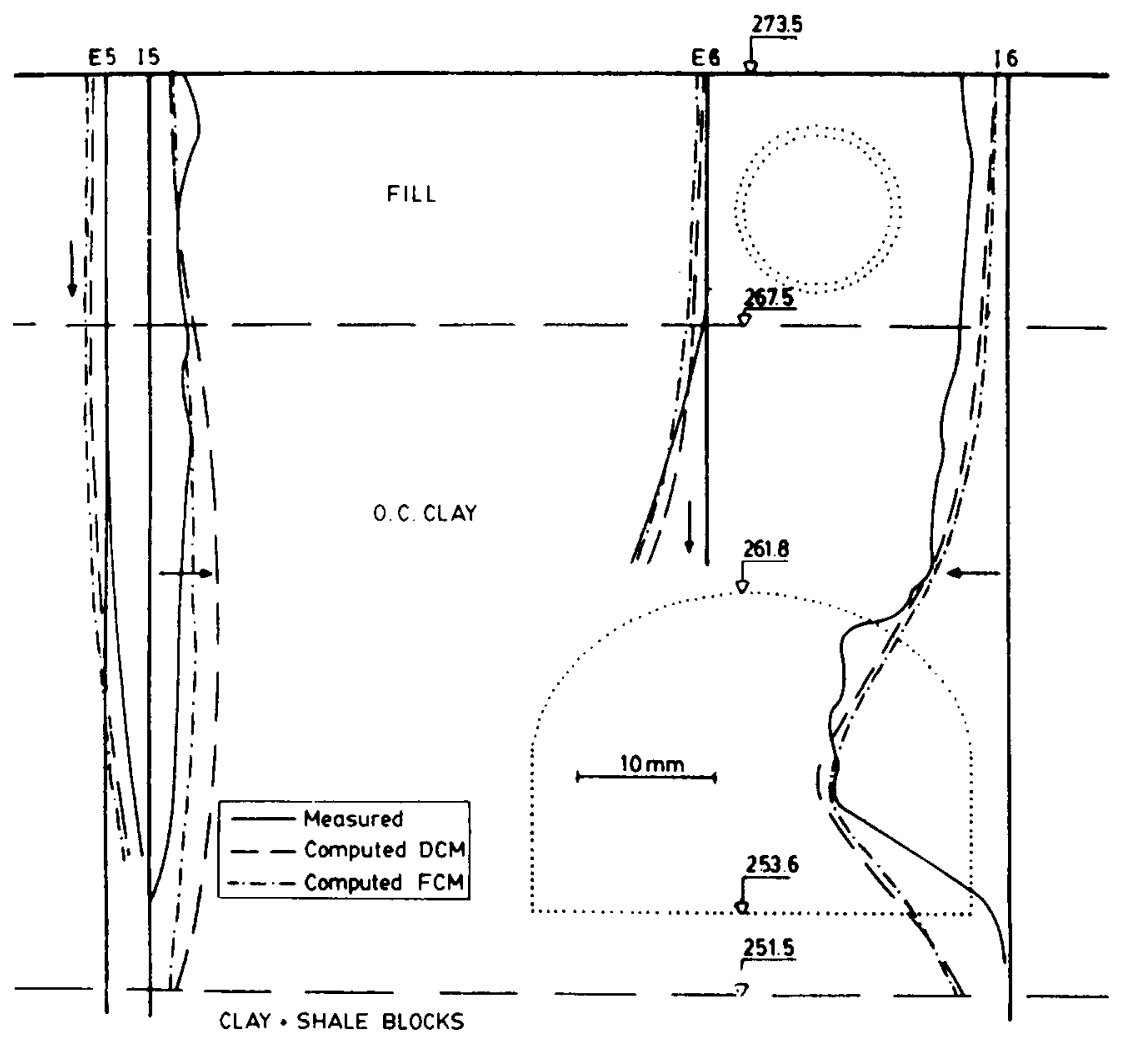

Fig. 11. Comparison between measured and computed displacements in section C. Least squares and maximum likelihood analyses. 
agreement between distributions of movements is quite satisfactory, given the uncertainties involved in the computations. In particular, the distribution of movements predicted with the set of parameters obtained from the maximum likelihood approach is somewhat closer to the measured one. Of course, the similarity of the geometry in sections B and C helps to reduce the importance of the structure of the model and contribute to the agreement between observed and calculated displacements.

\section{CONCLUDING REMARKS}

In this paper, an application of an identification of parameters methodology to a real engineering case is presented. This case corresponds to the characterization of the geological materials involved in the excavation of a tunnel near Barcelona (Spain).

The mathematical formulation of the procedure has been described in a companion paper [1]. The formulation is based on the maximum likelihood criterion which includes the least squares criterion as a particular case. Both criteria have been applied to the estimation of the mechanical properties of the materials involved.

A linear elastic isotropic model has been assumed to characterize the behaviour of the materials. The Young's modulus of three layers and the $K_{0}$ parameter in the excavation zone have been identified.

Reasonable agreement has also been obtained between the stiffnesses estimated from field measurement and those measured in the laboratory. In spite of the fact that the actual non-linearity of the material has not been modelled, the estimated parameters have been able to provide good predictions for the movements measured at another section of the tunnel. The identification procedure has also provided important information on the reliability of the estimated parameters, and on the structure of the models and the interdependency of the selected measurement points.

The case described has demonstrated the capability of the proposed backanalysis procedure for obtaining parameters in a geotechnical engineering problem.

\section{ACKNOWLEDGEMENTS}

The assistance of the "Departament de Política Territorial i Obres Públiques" of the Generalitat of Catalunya is gratefully acknowledged. 


\section{REFERENCES}

1. Ledesma, A., Gens, A. \& Alonso, E. E., Estimation of parameters in geotechnical backanalysis - I. Maximum likelihood approach. Computers and Geotech., 18 (1996) 1-27.

2. Wiggins, R. A., The general linear inverse problem: implication of surface waves and free oscillations for earth structure. Rev. Geophys. Space Phys., 10 (1972) 251-285.

3. Marquardt, D. W., An algorithm for least-squares estimation of nonlinear parameters. J. Soc. Ind. Appl. Math., 11 (1963) 431-441.

4. Ledesma, A., Identificación de parámetros en Geotecnia. Aplicación a la excavación de túneles. Ph.D. thesis, Technical University of Catalonia, Spain, 1987. 